\title{
Cross-clade simultaneous HIV drug resistance genotyping for reverse transcriptase, protease, and integrase inhibitor mutations by lllumina MiSeq
}

Dawn M Dudley ${ }^{1}$, Adam L Bailey ${ }^{1}$, Shruti H Mehta ${ }^{2}$, Austin L Hughes ${ }^{3}$, Gregory D Kirk ${ }^{4}$, Ryan P Westergaard ${ }^{5}$ and David $\mathrm{H} \mathrm{O}^{\prime}$ Connor ${ }^{1^{*}}$

\begin{abstract}
Background: Viral resistance to antiretroviral therapy threatens our best methods to control and prevent HIV infection. Current drug resistance genotyping methods are costly, optimized for subtype B virus, and primarily detect resistance mutations to protease and reverse transcriptase inhibitors. With the increasing use of integrase inhibitors in first-line therapies, monitoring for integrase inhibitor drug resistance mutations is a priority. We designed a universal primer pair to PCR amplify all major group M HIV-1 viruses for genotyping using Illumina MiSeq to simultaneously detect drug resistance mutations associated with protease, nucleoside reverse transcriptase, non-nucleoside reverse transcriptase, and integrase inhibitors.

Results: A universal primer pair targeting the HIV pol gene was used to successfully PCR amplify HIV isolates representing subtypes $A, B, C, D, C R F 01 \_A E$ and CRF02_AG. The universal primers were then tested on 62 samples from a US cohort of injection drug users failing treatment after release from prison. 94\% of the samples were successfully genotyped for known drug resistance mutations in the protease, reverse transcriptase and integrase gene products. Control experiments demonstrate that mutations present at $\geq 2 \%$ frequency are reliably detected and above the threshold of error for this method. New drug resistance mutations not found in the baseline sample were identified in $54 \%$ of the patient samples after treatment failure. $86 \%$ of patients with major drug resistance mutations had 1 or more mutations associated with drug resistance to the treatment regimen at the time point of treatment failure. 59\% of the emerging mutations were found at frequencies between $2 \%$ and $20 \%$ of the total sequences generated, below the estimated limit of detection of current FDA-approved genotyping techniques. Primary plasma samples with viral loads as low as 799 copies/ml were successfully genotyped using this method.
\end{abstract}

Conclusions: Here we present an Illumina MiSeq-based HIV drug resistance genotyping assay. Our data suggests that this universal assay works across all major group M HIV-1 subtypes and identifies all drug resistance mutations in the pol gene known to confer resistance to protease, reverse transcriptase and integrase inhibitors. This high-throughput and sensitive assay could significantly improve access to drug resistance genotyping worldwide.

Keywords: Human immunodeficiency virus, Antiretroviral therapy, HIV drug resistance, Drug resistance genotyping, Illumina MiSeq, Integrase inhibitor genotyping, Deep sequencing

\footnotetext{
* Correspondence: dhoconno@wisc.edu

'Department of Pathology and Laboratory Medicine, University of Wisconsin

School of Medicine and Public Health, Madison, WI, USA

Full list of author information is available at the end of the article
}

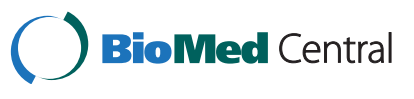

(c) 2014 Dudley et al.; licensee BioMed Central. This is an Open Access article distributed under the terms of the Creative Commons Attribution License (http://creativecommons.org/licenses/by/4.0), which permits unrestricted use, distribution, and reproduction in any medium, provided the original work is properly credited. The Creative Commons Public Domain Dedication waiver (http://creativecommons.org/publicdomain/zero/1.0/) applies to the data made available in this article, unless otherwise stated. 


\section{Background}

Antiretroviral therapy has been shown to be the most potent intervention for both treating and preventing HIV [1,2]. However, the effectiveness of antiretroviral drugs is threatened by the development and transmission of drug resistant virus [3,4]. HIV drug resistance genotyping is a clinically important tool to detect the emergence of viral resistance and maximize the benefit of current treatment options [5,6]. New sequencing techniques can improve sensitivity of drug resistance genotyping while also reducing costs. We recently published a paper outlining the benefits of using Roche/454 pyrosequencing to detect HIV drug resistance mutations with a capacity to reduce costs by 5 -fold [7]. As sequencing technologies have continually improved, the genotyping methods used in clinical settings to detect drug resistance have failed to evolve at a comparable pace. Here we present an updated deep sequencing genotyping assay utilizing the Illumina MiSeq. Advantages of this novel method over our Roche/454 approach include fewer systemic basecalling sequencing errors, increased multiplexing, use of a FDA-approved sequencing platform, detection of drug resistance to integrase inhibitors in addition to reverse transcription inhibitors and protease inhibitors with a single amplicon, and finally, simultaneous genotyping of all major group M HIV subtypes with a single set of amplification primers.

Detection of Integrase inhibitor drug resistance is particularly noteable because integrase inhibitors are now recommended by the department of Health and Human Services as part of first-line antiretroviral therapy in the United States [8]. The use of integrase inhibitors as part of initial antiretroviral therapy is expected to increase because of the recent FDA approval of a new once daily integrase inhibitor (elvitegravir) that can be co-formulated with emtricitabine, tenofovir disoproxyl fumarate and cobicistat as a single pill once daily regimen (STRIBILD) [9]. Current genotyping methods used to detect drug resistance to integrase inhibitors are limited and typically involve a separate assay from that used to identify drug resistance to reverse transcriptase and protease inhibitors $[10,11]$. However, due to proximity of the integrase protein-coding region to the reverse transcriptase and protease protein-coding region in the viral genome, it is feasible to incorporate drug resistance genotyping of all three gene products together from a single PCR amplicon. By using a single amplicon, no additional labor or expense is added to the assay already detecting protease and reverse transcriptase drug resistance mutations.

The FDA-approved HIV drug resistance genotyping assays currently in use were developed using HIV group $\mathrm{M}$ subtype $B$ viruses [12]. However, subtype $C$ comprises about half of the worldwide epidemic followed by subtype A (12\%) and then B (11\%) [13]. Current commercial genotyping assays perform rather poorly with non-subtype B viruses because seven sets of sequencing primers must bind to highly variable targets during sequencing [12,14]. Therefore, non- $\mathrm{B}$ assays require more replication and for many samples, fail altogether with the current commercially available genotyping assays. There have been many successful adaptations of the commercial Sanger-based genotyping assay to improve genotyping of non-B HIV subtypes, however, these assays do not include detection of integrase inhibitor mutations and are less sensitive than next-generation sequencing approaches [15-21].

Cost is a major barrier to drug resistance genotyping in resource-limited settings. Deep sequencing technologies have significantly reduced the cost of sequencing but are not yet incorporated into standard HIV drug resistance genotyping. An Illumina MiSeq sequencer can yield over 25 million sequencing reads per run with the latest technology (MiSeq reagent kit v3). Through multiplexing, 96 samples can be processed in one run, bringing the cost of sequencing alone to $<\$ 10 /$ sample. This is 3 -fold less than Sanger-based sequencing. In addition, the clonal nature of deep sequencing methods along with the large number of sequencing reads generated improves sensitivity for the detection of mutations compared to Sanger-based sequencing $[12,22,23]$. Lastly, the Illumina MiSeq platform is currently the least error-prone deep sequencing method $[24,25]$.

\section{Results and discussion}

\section{PCR primers amplify major group M HIV subtypes $A, B, C$,} D, CRF01_AE and CRF02_AG

HIV sequences from all subtypes found in the Los Alamos National Labs HIV database (http://www.hiv.lanl.gov) were used to design a universal primer set to amplify all major HIV subtypes as described in the Methods section (HIV2252-F and HIV5073-R). We obtained HIV isolates from the International Panel of $60 \mathrm{HIV}-1$ isolates from the NIH AIDS Research and Reference Reagent Program, Division of AIDS, NIAID, NIH (cat. \#11412) representing subtypes A, B, C, D, CRF01_AE and CRF02_AG. We selected 19 isolates to represent geographically diverse regions as shown in Table 1, isolated viral RNA and performed one step RT-PCR to create PCR amplicons according to the protocol outlined in the Methods section. Despite a wide range of TCID50 and/or P24 content in the panel isolates tested, all isolates yielded a strong amplification product after 40 cycles of PCR as visualized on a $1 \%$ agarose gel (Additional file 1). These results suggest that the primer pair designed for this method could be used to amplify all major group M HIV subtypes.

\section{Error associated with the genotyping assay}

To assess the error associated with the RT-PCR amplification and sequencing of samples, a clonal virus stock was generated from a plasmid containing the HXB2 
Table 1 HIV-1 subtypes tested with the universal primer set

\begin{tabular}{|c|c|c|c|c|}
\hline Virus & Subtype & $\mathrm{TCID} / \mathrm{mL}^{*}$ & $\mathrm{P} 24 \mathrm{ng} / \mathrm{mL}^{*}$ & RT-PCR \\
\hline 92UG_029 & $A$ & $1.26 \times 10^{5}$ & 150 & + \\
\hline 93RW_024 & A & NA & NA & + \\
\hline 00KE-KER2008 & A & $10^{2.60}$ & 57 & + \\
\hline 84US_MNp & B & $1.02 \times 10^{4}$ & 634 & + \\
\hline 85US_BA-L & B & $5.62 \times 10^{6}$ & 39.3 & + \\
\hline 91US_1 & B & $10^{5.0}$ & 294 & + \\
\hline 94US_33931N & B & $10^{3.10}$ & 235 & + \\
\hline 00TZ_A246 & C & $1.45 \times 10^{4}$ & NA & + \\
\hline 02ET_14 & C & $10^{4.10}$ & 12.2 & + \\
\hline 94IN_20635-4 & C & NA & NA & + \\
\hline 90SE_364 & C & $10^{6.0}$ & 157 & + \\
\hline 98US_MSC5016 & C & $10^{3.85}$ & 11.5 & + \\
\hline 93UG_065 & D & NA & NA & + \\
\hline 98UG_57128 & D & $10^{2.39}$ & 13 & + \\
\hline 99UG_A03349M1 & $\mathrm{D}$ & $2.05 \times 10^{4}$ & 290 & + \\
\hline 90TH_CM244 & CRF01_AE & $4.1 \times 10^{4}$ & 88 & + \\
\hline 97TH_NP1525 & CRF01_AE & $7.11 \times 10^{3}$ & 90 & + \\
\hline 91DJ_263 & CRF02_AG & $2.3 \times 10^{4}$ & NA & + \\
\hline 98US_MSC5007 & CRF02_AG & $4 \times 10^{4}$ & 127 & + \\
\hline
\end{tabular}

* Provided by the NIH AIDS Research and Reference Reagent Program.

HIV sequence. We PCR amplified and sequenced the stock as described in the Methods section and as depicted in Figure 1. We assessed all nucleotide variants present above a $0.1 \%$ frequency across the entire Pol region sequenced from two independent amplifications and sequencing runs of the HXB2 clonal stock amplicons. Any variant found in our clonal virus stock is expected to be due to PCR or sequencing errors. The frequency of these variants determined our error limit threshold for this assay. Nucleotide polymorphisms that result in a non-synonymous amino acid changes are presented in Additional file 2. Most nucleotide variants were present at frequencies less than $0.4 \%$, however, two regions contained variants at frequencies between $0.6 \%$ and $0.9 \%$. One insertion was present at a frequency of $0.9 \%$ that was associated with the end of the first of two back-to-back long homopolymer runs (AAAAAAGAAAAAA) found near the K101 and K103 drug resistance mutations, but not within them. Another single nucleotide polymorphism was found at a $0.6-0.7 \%$ frequency downstream of the integrase drug resistance mutations (nucleotide position 2779 (R275R in integrase)). This polymorphism was a synonymous change that did not alter the amino acid sequence. These results suggest that we see some evidence of increased mutations associated with homopolymers in Illumina data, as has been published, but that those mutations are not present above a $1 \%$ frequency nor do they impact the codons directly associated with drug resistance [26]. In addition, errors associated with the GGC sequence motif were also not found above a frequency of $0.5 \%$. We also detected five variants present at $99.9-100 \%$ frequencies that are likely differences between the modified HXB2 plasmid we sequenced and the published HXB2 strain and were ignored as variants caused by PCR or sequencing errors (non-synonymous changes shown in Additional file 2). Overall, no variants were found above a $0.9 \%$ frequency in our clonal HIV stock.

When specifically assessing nucleotide variants associated with amino acid changes linked to HIV drug resistance across the pol gene, we found that all variants within drug resistance sites were present at frequencies below $0.3 \%$ of the viruses sampled from our clonal stock (Additional file 2). Therefore, given both the error limits within the drug resistance sites and outside of the drug resistance sites, variants found at frequencies greater than $1 \%$, are likely authentic, while those below this threshold may result from RT-PCR and sequencing artifacts. Repeated sequencing of a subset of samples revealed that variants above $2.0 \%$ are consistently detected, while some variants found at frequencies between $1.0 \%$ and $2.0 \%$ are not (Additional file 2). Therefore, we designated $2.0 \%$ as the minimum threshold frequency for variants in subsequent experiments. Previous studies suggest that incorrect nucleotide incorporation associated with PCR error typically occurs at rates under $2.0 \%$, supporting our finding that variants found at a greater than $2.0 \%$ frequency are likely true variants [27]. Also, the error rates described here are very specific to the precise protocol, polymerases and sequencing kit used in this manuscript. Error rates should be reassessed if any changes to the method are made.

To determine whether nested PCR increased the error associated with low-frequency mutations, we performed the same test as described above, but subjected the clonal virus stock to two rounds of PCR under the same conditions used for primary samples. Once again, variants from the expected HXB2 sequence were present below a frequency of 1.0\% (Additional file 2).

We previously published a high throughput nextgeneration-based HIV drug resistance genotyping technique based on the Roche/454 sequencing platform [7]. One of the disadvantages of this system was the error associated with nucleotide homopolymer runs resulting from the sequencing chemistry used in the Roche/454 system. Sequence regions with homopolymer runs of three or more nucleotides are often miscalled, presenting a problem for some important drug resistance mutations like K103N and K65R that are encoded by homopolymeric nucleotide sequences. The competitive addition of reversible terminators used in Illumina's sequencing technology greatly reduces homopolymer errors resulting in more 


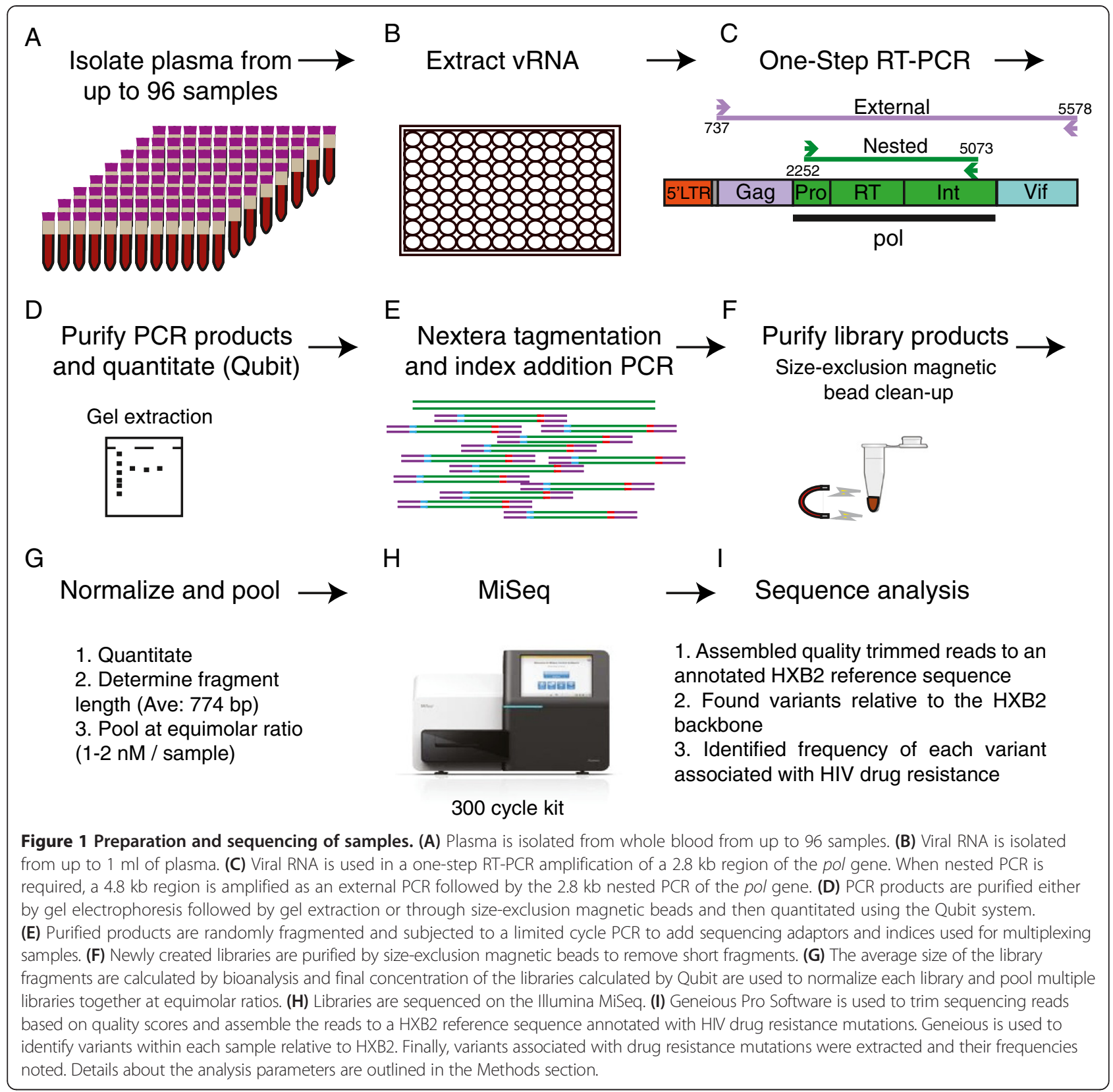

accurate identification of drug resistance mutations within these important sites. After trimming, the nucleotides representing the homopolymers in K103N and K65R mutations found in our patient samples maintained Phred quality scores $>\mathrm{Q} 30$ (or $\mathrm{p}=0.001$ ), lending greater confidence to the nucleotide bases called in these regions than was afforded by the Roche/454 assay where quality scores in these regions often dropped below Q20.

\section{PCR amplification using specimens from ALIVE}

We obtained plasma samples collected from adults with a history of injection drug use who participated in the AIDS Linked to the IntraVenous Experience (ALIVE) study in
Baltimore, MD [28]. Previous research with this cohort showed that virologic failure occurs with high frequency when participants experience incarceration, but it is not known whether the viral rebound that occurs in this setting is associated with development of drug resistance [29]. To answer this question while testing our method on primary HIV isolates, we genotyped HIV RNA from stored plasma specimens obtained from ALIVE participants. First, we isolated plasma viral RNA from 29 patients with samples from at least two time points for a total of 62 samples (see Additional file 3). 94\% (58/62) of the samples were successfully amplified and sequenced, though 9 of these samples required nested 
PCR amplification. Most of the samples that either failed or required nested PCR either had low viral load values $(<1,000 \mathrm{copies} / \mathrm{ml})$ or were $5-13$ years old and underwent multiple freeze-thaw cycles prior to PCR amplification (see Additional file 3). Of the samples that were successfully sequenced with viral loads above 2,000 copies $/ \mathrm{ml}$, 94\% (48/51) of the samples amplified with one round of PCR using the universal primer set. Since each unique patient sample amplified in at least one time point and our primers are universal for multiple subtypes, we do not believe that the subtype of the patient virus was the reason for the failure to amplify in three samples. In any event, our sequences clustered with subtype B virus in phylogenetic analysis of the pol gene sequences (Additional file 4). Overall, we expect that if this method was used on recently collected samples with viral loads greater than 2,000 copies/ml for timely genotyping assays, nested PCR would not be required. Lastly, we were able to sequence primary isolate samples with a viral load as low as 799 copies/ml with nested PCR and 1070 copies/ml with a single round of PCR (see Additional file 3). However, each patient sample likely exhibits a unique limit of detection depending on the sequence concordance between the primers and patient virus.

\section{Sequencing using specimens from ALIVE}

Including controls, 63 PCR amplicons were fragmented and pooled together in a single MiSeq run. The cluster density of the sequencing run was $663 \mathrm{k} / \mathrm{mm}^{2}$ and the average number of sequence reads obtained per sample was 146,780 (range: 74,848-244,428, stdev: 34,556 ). The coverage, or number of sequences that represent each drug resistance site after mapping to a reference sequence, varied between each patient and across the pol gene. Figure 2 shows the coverage of a representative sample

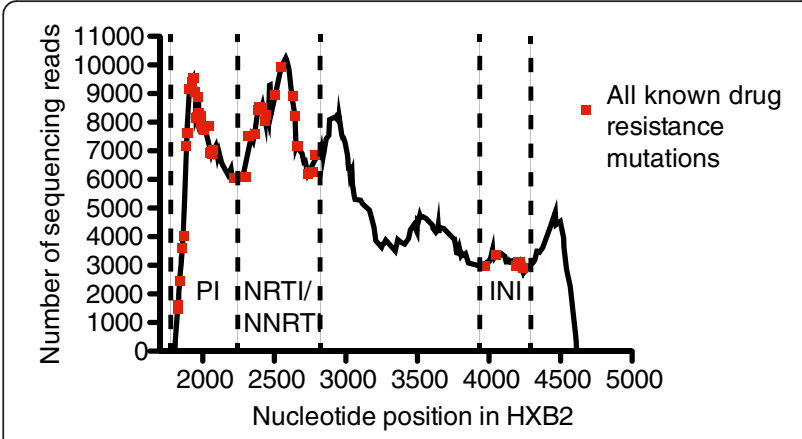

Figure $\mathbf{2}$ Representative sequence coverage across the pol gene. The number of sequences representing each nucleotide across the pol gene (i.e. "coverage") is shown for all sites that differed from HXB2 in a representative sample (sample 16 v11). Sites of all known drug resistance mutations are over-layed as red squares and the graph is divided into sections representing mutations associated with protease, reverse transcriptase or integrase drug resistance for reference. (patient 16) with 142,169 total reads. All nucleotides present between the first protease inhibitor resistance mutation (HXB2 position 1826) and last integrase inhibitor drug resistance mutation (HXB2 position 4238) were represented by more than 1,000 sequence reads in this patient (red squares in Figure 2). The goal of this method to generate a depth of coverage of $>1000$ sequencing reads/nucleotide position and in fact, at the major drug resistance sites, the average coverage of each mutation was over 1000 sequence reads (Table 2). Based on previous next-generation sequencing work, to accurately detect mutations at a frequency of $1.0 \%$, minimal coverage requirements have ranged from 300 to 1850 sequencing reads [22,30-32]. With our minimum frequency threshold set to $2.0 \%$, requiring a minimum of 1000 sequencing reads is conservative relative to previous publications. Across all samples, the average number of sequences representing each drug resistance site was 3,795 sequences. While coverage varied across the pol gene, the quality of all assembled sequences was very high due to the stringent trimming parameters (see Methods section) used during analysis.to remove nucleotides whose base call was deemed less than $99.9 \%$ accurate by the sequencing software prior to assembly (phred quality score $>$ Q30 or $\mathrm{p}>0.001$ ). Note that this data was generated prior to the release of the latest MiSeq sequencing kits, which now have an average output capacity of 25 million reads per sequencing run. This would more than double the reads obtained per sample as well as double the sequence read coverage at each nucleotide site given the same pooling strategy.

In general, across all patient samples and as exemplified in Figure 2, the coverage of the nucleotides associated with the protease inhibitor mutations found closest to the $5^{\prime}$ end of protease were lower than the number of sequences representing all other drug resistance mutation sites downstream. There are four accessory mutations (L10, V11, G16E and K20) and zero major mutations that sit close to the end of the original pol gene PCR amplicon that are sometimes affected by this reduced coverage (Table 2). These four sites are less represented because transposon-based fragmentation creates fewer fragments at the ends of the input DNA strand (original full-length pol gene amplicon) that have the necessary adaptor required for sequencing. Instead of 1000s of reads, sometimes these mutations are represented by hundreds of reads in this cohort (see Table 2). For the two mutations (L10 and V11) in our cohort that are represented by an average of 300 sequencing reads, a minimum frequency requirement could be increased to $6.5 \%$ to ensure that at least 20 reads are found containing the mutation, keeping the requirement the same as a mutation found at a $2 \%$ frequency in 1000 sequencing reads. This caveat to this approach could be solved by generating a primer that sits 
Table 2 Average sequencing read coverage for all drug resistance mutations identified in the ALIVE cohort

\begin{tabular}{|c|c|c|}
\hline Gene product & $\begin{array}{l}\text { Drug resistance } \\
\text { mutation* }\end{array}$ & $\begin{array}{l}\text { Ave. sequencing read } \\
\text { coverage }\end{array}$ \\
\hline \multirow[t]{22}{*}{ PR } & L10IFVC & 297 \\
\hline & V111 & 294 \\
\hline & G16E & 1022 \\
\hline & K20RMITV & 1917 \\
\hline & L24I & 1039 \\
\hline & D30N & 2169 \\
\hline & L33FIV & 3140 \\
\hline & M36LIV & 3639 \\
\hline & M46I/L & 2609 \\
\hline & I50L/V & 3895 \\
\hline & F53LY & 1213 \\
\hline & I54VTALM & 2122 \\
\hline & D60E & 3065 \\
\hline & $162 \mathrm{~V}$ & 3198 \\
\hline & L63P & 3346 \\
\hline & I64LMV & 2635 \\
\hline & A71VITL & 3800 \\
\hline & G73CSTA & 3045 \\
\hline & V82ATFSL & 3300 \\
\hline & L89VIM & 4199 \\
\hline & L90M & 4982 \\
\hline & I93LM & 5850 \\
\hline \multirow[t]{15}{*}{ RT } & M41L & 5014 \\
\hline & A62V & 4941 \\
\hline & K70R & 4324 \\
\hline & V75I & 10460 \\
\hline & V77I & 4787 \\
\hline & V90l & 4177 \\
\hline & K103N & 6048 \\
\hline & V106A/M/I & 6492 \\
\hline & V108I & 7424 \\
\hline & E138KAGQR & 7401 \\
\hline & M184V/I & 5906 \\
\hline & Y188LHC & 5283 \\
\hline & G190ASE & 4708 \\
\hline & $\mathrm{P} 225 \mathrm{H}$ & 5764 \\
\hline & M230LI & 8668 \\
\hline IN & S147G & 3123 \\
\hline
\end{tabular}

*Boldface mutations are major drug resistance mutations, non-boldface mutations are accessory mutations.

further upstream in the protease region, however, primers design is constrained when trying to keep the primers universal across multiple HIV clades. As mentioned above, with the latest MiSeq sequencing kits, coverage will also double at these sites. Lastly, reducing the multiplexing would also yield greater coverage per sample at this site if coverage of these mutations was a concern. The data from this patient cohort shows that this method combining 63 samples together results in characterization of major drug resistance mutations with thousands of sequencing reads representing most mutations. Based on our average coverage of 3,795 sequences, extrapolation would suggest that 96 samples could be pooled together and average coverage of major drug resistance mutations would still be over 1,000 sequences per drug resistance site when optimal cluster density is reached on the MiSeq sequencing chip. This would further increase by at least 2 -fold by using the latest MiSeq reagent kit V3 technology.

Although transposon-based fragmentation is theoretically unbiased, we observed consistently greater coverage in the reverse transcriptase protein coding region than protease and integrase coding regions across all samples, as exemplified in Figure 2. This similar coverage pattern between different samples over the same genetic regions is similar to that previously observed with whole genome HIV and SIV sequencing using the same fragmentation method [33]. Therefore, it is likely that there is some bias in fragmentation that yields ideally sized fragments for sequencing in some parts of the HIV genome over others.

Lastly, the assembled sequences were aligned and a maximum likelihood phylogenetic analysis was performed along with reference sequences from the panel of HIV isolates that we PCR amplified with our universal primer set (Additional file 4). As shown in Additional file 4, all samples from this cohort cluster with subtype B viruses, including the HXB2 control samples that we sequenced. In addition, the two time points from each sample clustered with each other and not with other samples, indicating that there was no cross-contamination resulting in the changes in drug resistance mutations found between the two time points from each patient sample.

\section{Drug resistance mutations detected in specimens from ALIVE}

To detect HIV drug resistance mutations in virus from the 29 ALIVE participants failing treatment after release from prison/jail, we analyzed the pol gene sequencing reads from two time points. First, the ALIVE participants successfully suppressed HIV before incarceration by using antiretroviral therapy. The first time point we sampled was at the time point just prior to this viral suppression. Some patients were drug naïve at this time point, but others were previously drug experienced (as outlined in Additional file 3) and either changed regimens or began taking ARTs after a break in treatment to reach viral suppression at the subsequent time point. The second time point represents the virus after treatment failure, 
as detected at an ALIVE study visit, after release from jail or prison. We obtained sequence data from both time points from 26/29 specimens. Relative to the first time point sequenced (baseline), new drug resistance mutations emerged in $54 \%(14 / 26)$ of the patients exhibiting treatment failure that were not detected at baseline (Figure 3A and Table 3). One additional sample (patient 27) contained major drug resistance mutations at both time points tested that may have contributed to treatment failure at the second time point (Table 4). These mutations, I54V, L90M, $\mathrm{K} 103 \mathrm{~N}$ and $\mathrm{M} 184 \mathrm{~V}$, were present at frequencies $>85 \%$ at both time points with most being present at $>99 \%$ of sequences. The remaining samples without emerging drug resistance mutations contained primarily accessory mutations at baseline or at the second time point or very low level major drug resistance mutations that disappeared by the second time point and therefore likely do not contribute to treatment failure. Therefore, these patients may be failing treatment for reasons other than drug resistance mutations in the pol gene product. For example, some patients may fail due to lack of adherence or presence of mutations in Gag or the cytoplasmic tail of Env, which have been recently reported to affect drug resistance to protease inhibitors [34-36]. In the future it will be possible to expand our technique to include these regions outside of the pol gene, however, it would require amplification of more than one amplicon prior to fragmentation and sequencing.

\section{Association of major drug resistance mutations with treatment in ALIVE}

As a way to assess our drug resistance genotyping assay, we compared the major drug resistance mutations identified by our genotyping assay with the treatment regimens reported by the ALIVE study participants at the time of treatment failure. We define major drug resistance mutations as those included in the Stanford Drug Resistance Database matrices that are associated with high levels of phenotypic drug resistance [37,38]. This is opposed to accessory mutations whose role in treatment failure is less clear. Overall, 57\% (8/14) of the patients with virus harboring any emerging drug resistance mutations relative to the first time point tested had major drug resistance mutations (Figure 3A and Table 3). We focused our mutation/treatment association analysis on these eight patients and analyzed the major mutations found in their virus using the Stanford drug resistance database Genotype Resistance Interpretation calculator $[37,38]$. The results of the calculator were then compared to each patient's self-reported treatment regimen at the time of failure (Table 4). Of the eight patients harboring virus with major drug resistance mutations, seven patients had virus with mutations associated with a high level of drug resistance to one or more of the drugs

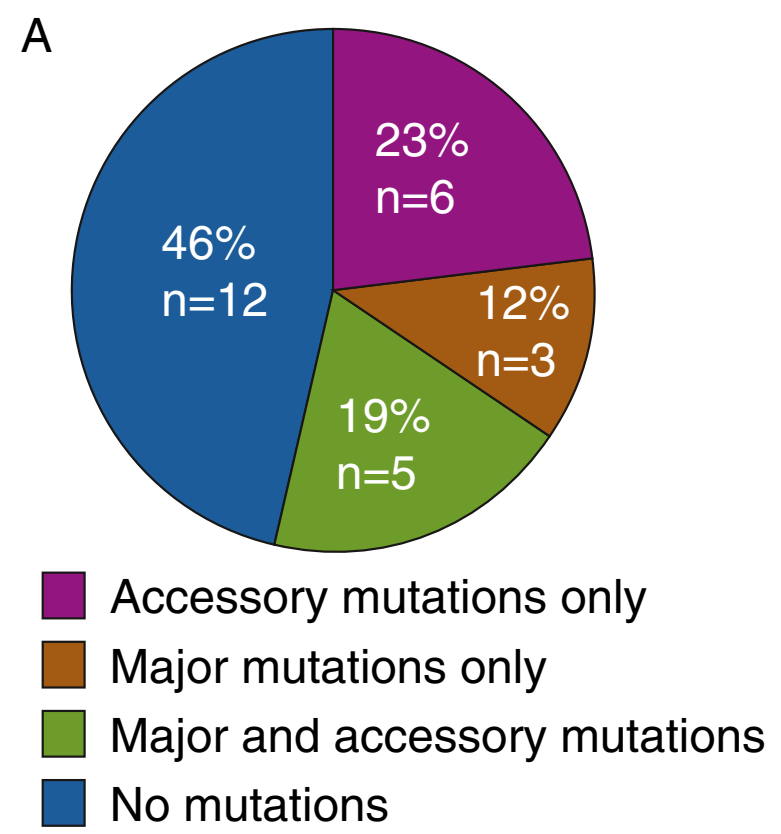

B

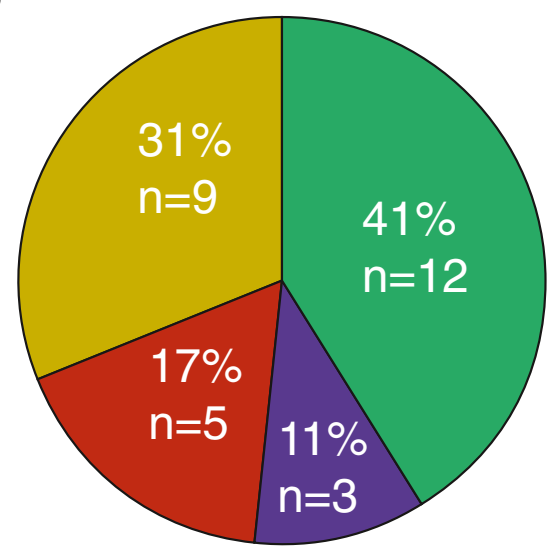

Accessory mutations $<20 \%$
Accessory mutations $>20 \%$
Major mutations $<20 \%$
Major mutations $>20 \%$

Figure 3 Characterization of the drug resistance mutations identified in the ALIVE cohort after treatment failure. (A) The frequency of different types of HIV drug resistance mutations found in 26 patient samples with after treatment failure. Major mutations are as defined by the Stanford drug resistance database and accessory mutations are all other mutations associated with drug resistance as designated by the International AIDS Society (IAS). (B) Percentages of the 29 emerging mutations found at the second time point but not first time for all participant samples that were major or accessory mutations and whether the mutations rose above the $20 \%$ threshold expected to be detected by commercial HIV drug resistance genotyping techniques. 
Table 3 Drug resistance mutations emerging after incarceration in the ALIVE cohort

\begin{tabular}{|c|c|c|}
\hline Drug class & Mutation & Sample \#(s) (\% frequency) \\
\hline \multirow[t]{13}{*}{ Protease inhibitors } & L10IFVC & $19(7.9 \%)$ \\
\hline & V111 & $25(6.0 \%)$ \\
\hline & G16E & $25(3.2 \%)$ \\
\hline & K20RMITV & $24(7.0 \%)$ \\
\hline & M36LIV & $24(5.5 \%)$ \\
\hline & M46IL & $27(7.3 \%)$ \\
\hline & F53LY & $16(5.1 \%)$ \\
\hline & $162 \mathrm{~V}$ & $4(15.3 \%)$ \\
\hline & I64LMV & $4(13.7 \%)$ \\
\hline & G73CSTA & $6(8.1 \%)$ \\
\hline & V771 & $13(4.6 \%)$ \\
\hline & L89VIM & $22(3.6 \%)$ \\
\hline & L90M & $6(16.2 \%)$ \\
\hline \multirow[t]{3}{*}{ NRTI inhibitors } & M41L & $2(13.3 \%)$ \\
\hline & $\mathrm{A} 62 \mathrm{~V}$ & $2(3.1 \%)$ \\
\hline & M184V & $\begin{array}{l}2(99.9 \%), 5(82.8 \%), 9(99.8 \%), \\
10(100 \%), 16(56.6 \%), 23(68.5 \%)\end{array}$ \\
\hline \multirow[t]{4}{*}{ NNRTI inhibitors } & K103N & 5 (100\%), 10 (100\%), 23 (99.8\%) \\
\hline & V108l & $23(59.8 \%)$ \\
\hline & E138KAGQR & $16(7.6 \%)$ \\
\hline & $\mathrm{P} 225 \mathrm{H}$ & $23(55.3 \%), 10$ (99.9\%) \\
\hline Integrase inhibitors & S147G & $16(7.6 \%)$ \\
\hline
\end{tabular}

Boldface font $=$ major mutation. Regular font $=$ accessory mutation .

in their regimen at the time point of failure (boldface in Table 4). Table 4 also shows the mutational load (viral load"frequency of mutation) of each mutation within each patient. M184V and K103N were the major mutations associated with treatment failure in this cohort and it is notable that the major drug resistance mutations that correlate with the drug regimen at the point of treatment failure are found at frequencies well above $50 \%$, indicating drug selection pressure. As expected, the major mutations identified in most of the patients of this cohort using our genotyping technique associated well with the treatment regimens reported by the patients at the time of treatment failure.

Major drug resistance mutations emerging after treatment failure were then compared with all drugs self-reported for each individual during the course of all time points where data was collected. As shown in figure 3B, almost half $(14 / 29)$ of all the 29 mutations emerging after treatment failure were major mutations. Overall, 10/14 major drug resistance mutations were associated with the patient self-reported drug regimens from either the time point of failure or previous time points (Table 4). These mutations were found at frequencies $>50 \%$ while those that do not associate with the treatment regimen are found at $<20 \%$. Altogether, these data are consistent with the conclusion that this genotyping assay is detecting drug resistance mutations that would be expected based on the treatment regimens reported by the participants in the ALIVE cohort.

\section{Accessory drug resistance mutations detected in ALIVE}

Accessory mutations are those that alone may not render resistance, but may contribute to resistance when combined with other mutations. All mutations identified on the International AIDS Society (IAS) USA drug resistance mutation list that were not part of the Stanford drug resistance database major mutation matrices were categorized as accessory mutations in this study. We identified accessory mutations in $23 \%$ of ALIVE patient samples that did not have major drug resistance mutations at the time of treatment failure (Figure 3A). Most of these mutations were present at frequencies $<20 \%$ (Figure 3B and Table 3). While it is likely that these accessory mutations contributed to resistance, this cohort was not appropriately powered to explore the correlations between accessory mutations and drug resistance more definitively.

\section{Minority drug resistance variants in the ALIVE cohort}

Currently, FDA-approved Sanger sequencing-based HIV drug resistance genotyping methods such as ViroSeq identify mutations present above a frequency of $20 \%$ within a host's viral population [12,22]. Therefore, mutations found below this $20 \%$ frequency (i.e. minority variants) might be missed by current commercial techniques. As shown in figure 3B and detailed in Table 3, 59\% (17/29) of all drug resistance mutations found in the ALIVE cohort by this genotyping technique were below the $20 \%$ frequency limit of detection of current genotyping. This indicates that the presented genotyping technique is likely more sensitive than those currently available commercially. Development of sensitive assays, like the genotyping assay presented here, will support future studies designed to delineate which minority mutations are important prior to the start of specific antiretroviral therapy. In our cohort, mutations likely contributing to treatment failure of a given drug regimen were predominantly present at frequencies $>20 \%$.

An important consideration when detecting lower frequency mutations or variants from samples with lower viral loads is reliability of the variant frequency detected and possibility of template resampling. While we were unable to do this with the presented cohort due to sample limitations, PCR amplifying a sample in triplicate and assessing the variant frequencies of each independent test for samples of different viral loads would be an important reliability test if adapting this methodology to clinical samples. In addition, when the 
Table 4 Association between the major drug resistance mutations emerging in treatment failures from the ALIVE cohort, the drug regimens reported at the time of failure, and expected resistance profile based on genotype results

\begin{tabular}{|c|c|c|c|c|c|c|}
\hline $\begin{array}{l}\text { Sample \# } \\
\text { visit \# }\end{array}$ & $\begin{array}{l}\text { Drug resistance } \\
\text { mutation }\end{array}$ & $\begin{array}{l}\text { Gene } \\
\text { product }\end{array}$ & $\begin{array}{l}\text { Frequency of DR } \\
\text { mutation (coverage)* }\end{array}$ & $\begin{array}{l}\text { Mutational load } \\
\text { (copies/ml) }\end{array}$ & $\begin{array}{l}\text { Treatment at time } \\
\text { point of failure }\end{array}$ & $\begin{array}{l}\text { Stanford resistance profile } \\
\text { for major mutations }\end{array}$ \\
\hline \multirow[t]{2}{*}{$2 \vee 15$} & M41L & RT & $13.3 \%(5316)$ & 6913 & ZDV, зTC & $\mathrm{H}: \mathbf{3 T C}, \mathrm{FTC}$ \\
\hline & M184V & RT & $99.9 \%(7721)$ & 51926 & & $L: A B C, D D I$ \\
\hline \multirow[t]{4}{*}{$5 \vee 20$} & $\mathrm{~K} 103 \mathrm{~N}$ & RT & $100 \%(5807)$ & 513564 & ZDV, 3TC, & H: 3TC, FTC, \\
\hline & M184V & RT & $82.8 \%(5266)$ & 425231 & $A B C, E F V$ & EFV, NVP \\
\hline & & & & & & $L: A B C$ \\
\hline & & & & & & PL: DDI \\
\hline \multirow[t]{3}{*}{$6 \vee 20$} & $\angle 90 M$ & $\mathrm{Pl}$ & $16.2 \%(3602)$ & 179820 & TDF, FTC, DRVr, RAL & $\mathrm{H}: \mathrm{NFV}$ \\
\hline & & & & & & I: IDV, SQV \\
\hline & & & & & & L: ATV, LPV, FPV, \\
\hline \multirow[t]{3}{*}{$9 v 33$} & M184V & RT & $99.8 \%(7493)$ & 420158 & ZDV, 3TC, ABC, RTV, FTC & H: 3TC, FTC \\
\hline & & & & & & $L: A B C$ \\
\hline & & & & & & PL: DDI \\
\hline \multirow[t]{3}{*}{$10 \mathrm{v} 10$} & K103N & RT & $100 \%(5979)$ & 40200 & EFV, TDF, FTC & H: 3TC, FTC, EFV, NVP \\
\hline & M184V & RT & $100 \%(5399)$ & 40200 & & $L: A B C$ \\
\hline & & & & & & PL: DDI \\
\hline \multirow[t]{4}{*}{$16 \mathrm{v} 11$} & E138K & RT & $7.6 \%(8955)$ & 27149 & 3TC, RTV, LPV & H: 3TC, FTC \\
\hline & M184V & RT & $56.6 \%(8222)$ & 202190 & & I: RPV \\
\hline & S147G & IN & $7.0 \%(3123)$ & 25006 & & $L: A B C$ \\
\hline & & & & & & PL: DDI, EFV, ETR, NVP \\
\hline \multirow[t]{3}{*}{$23 \vee 17$} & $\mathrm{~K} 103 \mathrm{~N}$ & RT & $99.8 \%(6110)$ & 16168 & EFV, TDF, FTC & H: 3TC, FTC, EFV, NVP \\
\hline & M184V & RT & $67.1 \%(6182)$ & 10870 & & $L: A B C$ \\
\hline & & & & & & PL: DDI \\
\hline \multirow[t]{2}{*}{$27 \vee 05$} & M46I** & $\mathrm{Pl}$ & $7.3 \%(3591)$ & 1262 & $\mathrm{~d} 4 \mathrm{~T}, \mathbf{3 T C}, \mathbf{A B C}, \mathrm{IDVr}$ & L: NFV \\
\hline & & & & & & $\begin{array}{l}\text { PL: ATV, FPV, IDVr, LPV } \\
\text { (non-emerging DR related } \\
\text { to treatment: } \mathrm{H}: \mathbf{3 T C}, \mathrm{L}: \mathbf{A B C} \text { ) }\end{array}$ \\
\hline
\end{tabular}

Boldface: correlation between mutation, treatment and drug resistance profile in each patient sample at the time point of treatment failure. $\mathrm{H}=\mathrm{high}$ level drug resistance, I = intermediate drug resistance, $\mathrm{L}=$ low level drug resistance, $\mathrm{PL}$ : potential low level drug resistance. Drug resistance mutations in italics are not associated with any reported drug taken by the patients throughout the course of this study. *Coverage refers to the number of sequencing reads representing a given nucleotide. **Patient 27 virus also harbored I54V, L90M, K103N and M184V at both time points. These mutations did not emerge between treatment success and failure, but likely contributed to overall failure.

number of sequences representing each nucleotide variant exceeds the number of virus genome templates (due to low viral loads) used to initiate the RT-PCR, then the sequences are representing each template more than once. This could result in false variant frequencies if there is a bias for one template over another during the PCR amplification. One way to address this concern would be to require higher viral loads or multiplex more samples on the sequencer to reduce the number of sequencing reads to below the number of templates added to the initial RT-PCR reaction. In this study, therefore, it is possible that some of the frequencies presented for samples with lower viral loads are not entirely accurate due to possible template resampling (Additional file 3). It remains questionable exactly what it means to have a mutation present at $10 \%, 50 \%$ or $99 \%$. With our current understanding of drug resistance mutations, a mutation present at any frequency could quickly turn into a mutation present in nearly all viruses given drug selection pressure. Therefore, an important drug resistance mutation present at any frequency above the error rate would likely warrant recommendations for changes to drug regimens regardless of the actual frequency detected.

Lastly, one of the advantages of this MiSeq genotyping method includes detection of integrase inhibitor drug resistance mutations. One patient in the ALIVE cohort (patient 16) with no prior exposure to integrase inhibitors had a virus with a minority (7.6\%) drug resistance mutation to Elvitegravir (Tables 3 and 4). Such a mutation should be considered when assigning a treatment regimen to this individual already failing their current antiretroviral regimen at the time of sample collection. The rollout of 
integrase inhibitors as part of initial treatment regimens increases the need for integrase inhibitor drug resistance testing prior to treatment initiation.

\section{Comparison of Illumina MiSeq platform with other platforms}

We have already highlighted the advantages of the Illumina MiSeq platform over our previous work using the Roche/454 GS junior platform. Additionally, Roche plans to phase out 454 machines in 2016 making this platform obsolete for future development. This work is also conceptually similar to recent work from Gibson et al. describing comprehensive HIV drug resistance genotyping across multiple HIV group $M$ subtypes for protease, reverse transcriptase and integrase inhibitors as well as coreceptor tropism using the Ion Torrent Personal Genome Machine [31]. There are, however, specific advantages of our methodologies using the MiSeq, including greater output (Illumina MiSeq: 13-15Gb vs. Ion Torrent PGM: 1Gb) and reduced systemic sequencing error rates (Illumina MiSeq: $<0.4 \%$, Ion Torrent: $1.78 \%$ ) [25]. Fewer errors reduce analysis time and improve data reliability. In addition, our method is technically simpler, requiring a single fragmented PCR amplicon over the three required for the Gibson et al. method. Lastly, Illumina MiSeq is the first and only next-generation sequencing platform to receive FDA approval for assays developed on that device, making it the optimal deep sequencing platform to develop a HIV drug resistance assay for possible clinical use in the future [39].

\section{Data analysis considerations}

Data analysis of next-generation sequencing techniques can be exceptionally difficult and requires many considerations. For example, when assessing viral variants related to HIV drug resistance one must consider the viral load of the samples to assess the possibility of template resampling and its effects on frequency determination, error associated with PCR amplification, systematic errors associated with sequencing, and accurate data analysis.

One of the advantages of the described data analysis approach is its relative simplicity using Geneious software. This software is very user-friendly and does not require a strong background in bioinformatics or programming skills. The parameters used in this analysis are fully defined in the Methods section and briefly include 3 basic steps: (1) pair the paired-end sequences together for each sample (2) trim ends based on quality and reference map against an annotated HXB2 sequence available here: (https://dholk.primate.wisc.edu/wiki/dho/ public/page.view?name=default) (3) call variants relative to the HXB2 reference sequence. Steps 2 and 3 can be performed simultaneously for all samples in a sequencing run. Overall, only a few hours of hands-on time are necessary to complete steps 1 , initiate step 2 and initiate step 3, with several hours of hands-off time to complete steps 2 and 3 . Once the variants have been detected, the annotation table results can be exported out of Geneious and assessed in Microsoft Excel or in a text editor to determine which samples have which drug resistance mutations and their frequencies. Depending on the speed of the computer used to perform this analysis, a run of 64 samples could be fully analyzed within two days on a standard laptop with most of the analysis performed in Geneious being hands-off. A fully automated analysis approach would be required to use this method in a clinical setting, however, the provided methods are relatively quick and simple for research-based projects and is considerably faster than Sanger-based data analysis of 64 samples.

One of the sequence analysis challenges with working with HIV drug resistance is that resistance is defined by changes in amino acid sequence that could be rendered by several different nucleotide variants. Many analysis tools consider a single nucleotide variant at a time and not necessarily the effect of two nucleotide changes from a single sequence within a codon that might change an amino acid. For example, a variant caller might call a change from ATG (Met) to GTA (Val) as a mix of ATA (Ile) and GTG (Val), considering each single nucleotide change away from ATG as separate even though they are present in the same sequence. We have worked with the developers at Geneious to create a phased variant detection approach that is accurate when assessing nucleotide variants in the context of amino acid changes, which is paramount to our analysis approach. This variant detection approach is now built into the latest Geneious software release.

Lastly, while we can identify drug resistance mutations in the protease, reverse transcriptase and integrase regions simultaneously with this assay, given the nature of library formation and Illumina MiSeq sequencing we cannot necessarily associate together mutations found in one area of the pol gene product with mutations found in another area. With the paired-end technology it is possible to link one mutation from the same template to a mutation found $\sim 600$ bp downstream, but we cannot make those associations with mutations that span further apart. In this regard, this assay is not more informative than traditional genotyping assays using Sanger-based sequencing, which also cannot associate mutations together across multiple pol gene products. The only way to reliably span the entire pol gene from a single virus involves either cloning and sequencing or limiting dilution PCR and sequencing. However, sequencing technologies continue to improve and as error rates improve for techniques that sequence thousands of nucleotides in a single sequencing read, linking together all drug resistance mutations will become much easier. 


\section{Conclusions}

We have designed a universal primer set that amplifies part of the pol gene from the major HIV-1 subtypes A, B, C, D, CRF01_AE and CRF02_AG. These primers were used in an Illumina MiSeq-based sequencing method for the detection of drug resistance in primary HIV samples from individuals failing treatment after release from prison. We characterized drug resistance to protease, reverse transcriptase and integrase inhibitors simultaneously. $54 \%$ of the patients failing treatment harbored mutations in their virus at the time of treatment failure that were not present before treatment. Of the patients with virus harboring major drug resistance mutations, all but one patient had virus with mutations associated with resistance to the self-reported treatment regimen at the time of treatment failure. This finding suggests that virologic failure associated with incarceration may be due to the development of drug resistance mutations in about half of cases. This implies that actions to prevent drug resistance, such as preventing treatment interruption during and after incarceration, may reduce virologic failure associated with incarceration. $59 \%$ of all the mutations found were present at frequencies between $2 \%$ and $20 \%$ of the within-host virus population and would likely have been missed by traditional Sanger-based genotyping techniques. The technique presented in this manuscript should facilitate larger studies to elucidate the role(s) of these minority variants in treatment failure- an important consideration as genotyping moves to more sensitive sequencing platforms. Additionally, these methods can be adapted to sequence any region of the HIV virus by designing new primer sets to study a variety of questions related to HIV evolution. The results shown here suggest that the presented method is a significant advance over current HIV drug resistance genotyping by improving sensitivity, broadening the detection of mutations from protease through integrase, and improving genotyping in non-B HIV subtypes.

\section{Methods}

\section{Ethics statement}

The subjects used in this study provided informed written consent. The phlebotomy protocol and consent forms were approved by the Internal Review Board of Johns Hopkins University. The collected samples were de-identified and sent to the University of Wisconsin-Madison for sequencing. The protocol was approved by the Health Sciences Institutional Review Board at the University of WisconsinMadison (protocol number 2012-0140).

\section{Study subjects}

Sixty-two participant samples were selected for inclusion in this study. These samples originated from individuals with a history of injection drug use who participated in the ALIVE study between 1997 and 2009 [29,40-42]. Selected participants were successfully treated with antiretroviral therapy (ART) prior to incarceration, but failed ART after release from prison. A baseline, pre-incarceration blood sample collected at a study visit before viral suppression was achieved and a follow-up sample collected after release from prison and treatment failure were obtained for this study (Additional file 3). As outlined in Additional file 3, many study participants were not drug naïve at the first time point we genotyped, but either failed their drug regimen or stopped taking antiretroviral drugs leading to an increase in viral load at the time point we sampled. Either a change in regimen or restarting a drug regimen resulted in viral suppression at the time point following the first time point we sampled. However, at a subsequent time point that coincided with the release from prison, individuals failed treatment again, which is the second time point that we tested. The range of viral load of these samples was 799-1,110,000 copies/ml. Treatment regimens were self-reported by the patients at each time point a sample was collected.

\section{HIV viral stocks}

Viral stocks containing subtypes A, B, C, D, CRF01_AE, and CRF02_AG were obtained from the NIH AIDS Research and Reference Reagent Program, Division of AIDS, NIAID, NIH (cat. \#11412) and used to test the subtype specificity of our primers. Viruses were provided by Robert Gallo, Smita Kularni, UNAIDS, Nelson Michael, and Victoria Polonis. The pHXBn-PLAP-IRES-N+ plasmid (cat. \#3610) was obtained through the NIH AIDS research and reference reagent program, Division of AIDS, NIAID, NIH from Dr. Benjamin K. Chen and Dr. David Baltimore. To produce a clonal viral stock to assess error associated with our method, the pHXBnPLAP-IRES-N+ (HXBn) plasmid was transfected into $239 \mathrm{~T}$ packaging cells using the Xfect Transfection Reagent (Clontech). The supernatant was collected two days after transfection to limit virus production to a single round and the virus was quantitated using the Lenti-X qRT-PCR titration kit (Clontech). Viral RNA was isolated from this supernatant (viral load $=115,000$ copies $/ \mathrm{ml}$ ) and subjected to one-step RT-PCR and sequenced using the same protocol that was used to sequence patient samples. The initial plasmid DNA was also sequenced using the same protocol as the research samples without the initial reverse transcription step. Both DNA and viral RNA starting templates are expected to have no variants relative to the HXB2 reference sequence (GenBank NC_001802). In addition, we sequenced the protease and reverse transcriptase gene products from the HIV plasmid used to generate the clonal virus using a Sanger-based approach as a standard to verify the pol gene sequence in the plasmid. This verification revealed two mutations in (HXB2 positions 1805 and 2473) the plasmid that were not expected based on 
the published map of the vector and GenBank entry. These mutations in the plasmid were found in $99 \%$ of the viral RNA sequenced from the clonal virus stock and were ignored during analysis as changes in the plasmid relative to the published HXB2 sequence. All other variants found by Illumina sequencing in either the plasmid or the viral stock were considered "false" variants that likely resulted from error in the PCR, sequencing, or analysis of the sequences. We used the maximum frequency found as "false" variants $(0.9 \%)$ as the minimum frequency threshold for "real" variants found within a patient's viral population $(1.0 \%)$ that do not likely stem from error associated with the sequencing method.

\section{Primer design}

Sequences representing multiple HIV subtypes were downloaded from the Los Alamos National Laboratory HIV database and aligned using the software program Geneious version 5.6. Regions of the pol gene flanking all known drug resistance mutations found in the protease, reverse transcriptase, and integrase protein coding regions were searched for conservation between group M HIV isolates. Two regions containing high conservation were identified and used to design PCR primers. Primers were optimized for melting temperatures, GC content and reduced hairpin and/or primer dimer formation as much as possible given restraints of the conserved regions (NetPrimer, Premier Biosoft International). The sequences of these primers named HIV2252-F and HIV5073-R can be found in Additional file 5 (nested primers). The forward primer contains a mismatch in the middle of the primer for some subtypes and is therefore designed with a degenerate nucleotide at that position $(R=A / G)$. The reverse primer contained a mismatch in the middle of the sequence in some subtype $\mathrm{C}$ isolates. Two separate reverse primers were designed and tested with subtype $\mathrm{C}$ isolates. The primer that matched all group $\mathrm{M}$ isolates but contained a mismatch with some subtype $\mathrm{C}$ isolates amplified all subtypes well including all subtype $C$ viruses tested and was used in this study (HIV5073-R). The expected PCR product size is $2892 \mathrm{bp}$. The external primers, as shown in Additional file 5, were previously designed (Todd Allen, personal communication).

\section{Sample preparation and PCR amplification}

A schematic diagram representing the major steps to prepare samples for drug resistance genotyping using the method described in this paper is presented in Figure 1. Plasma from each sample was centrifuged at $14,000 \mathrm{x} \mathrm{rpm}$ at $4^{\circ} \mathrm{C}$ for 1 hour to concentrate virus for viral RNA extraction using the QIAamp Viral RNA Mini Kit (Qiagen). Viral RNA was subjected to one-step RT-PCR amplification using Superscript III Reverse Transcriptase and High Fidelity Platinum Taq Polymerase (Invitrogen) and the primers HIV2252-F and HIV5073-R. RT-PCR conditions were as follows: reverse transcription was performed at $50^{\circ} \mathrm{C}$ for $30 \mathrm{~min}$., the enzyme was denatured at $94^{\circ} \mathrm{C}$ for $2 \mathrm{~min}$., 40 cycles of amplification was performed at $94^{\circ} \mathrm{C}$ for 15 sec., $55^{\circ} \mathrm{C}$ for 30 sec., and $68^{\circ} \mathrm{C}$ for $3 \mathrm{~min}$., followed by a $68^{\circ} \mathrm{C}$ extension for 10 minutes. Amplification was more reliable with the addition of 5\% DMSO to the PCR reaction. Products were visualized on a DNA Flashgel (Lonza) and any sample containing PCR amplicons other than the product of interest was electrophoresed on a 1\% agarose gel and the expected $2.8 \mathrm{~kb}$ amplicon was extracted using a Qiagen gel extraction MinElute kit (Qiagen). All samples were further purified using size exclusion beads (Agilent XP) to remove primer dimers at a 1:1 DNA:bead (volume) ratio. Samples that failed to amplify with a single round of PCR were subjected to nested PCR using the following primers in an external PCR (Additional file 5): HIV737-F and HIV5578-R. External PCR conditions included reverse transcription at $50^{\circ} \mathrm{C}$ for $60 \mathrm{~min}$., polymerase activation at $94^{\circ} \mathrm{C}$ for $2 \mathrm{~min}$., 2 cycles of $94^{\circ} \mathrm{C}$ for $15 \mathrm{sec}$., $60^{\circ} \mathrm{C}$ for $30 \mathrm{sec}$., and $68^{\circ} \mathrm{C}$ for $4 \mathrm{~min}$. and 45 sec., 2 cycles of $94^{\circ} \mathrm{C}$ for 15 sec., $58^{\circ} \mathrm{C}$ for 30 sec., and $68^{\circ} \mathrm{C}$ for $4 \mathrm{~min}$. and $45 \mathrm{sec}$. 31 cycles of $94^{\circ} \mathrm{C}$ for $15 \mathrm{sec}$., $55^{\circ} \mathrm{C}$ for $30 \mathrm{sec}$, and $68^{\circ} \mathrm{C}$ for $4 \mathrm{~min}$. and $45 \mathrm{sec}$. followed by a $10 \mathrm{~min}$. extension at $68^{\circ} \mathrm{C} .5 \mu \mathrm{l}$ of the $25 \mu \mathrm{l}$ external PCR was used in a nested PCR using the conditions for single round PCR described above excluding the reverse transcription step. The nested primer set is shown in Additional file 5 and is the same primer set used for samples requiring only a single round of amplification.

\section{Amplicon fragmentation and sequencing}

Following size exclusion purification, PCR amplicons were quantitated using picogreen dye and the Qubit (Invitrogen) fluorometer or DTX 800 Multimode detector plate reader (Beckman Coulter). $1 \mathrm{ng}$ of each sample was fragmented using Nextera XT transposon technology (Epicentre). The average size of the fragmented PCR amplicons was 774 bp (range 519-1060 bp) as detected by bioanalysis (Agilent). Following Nextera tagmentation, Illumina sequencing adaptors and molecular tags were added via PCR, size exclusion bead purification was performed, and samples were quantitated using picogreen as previously described. Individual samples were then pooled at equimolar ratios (1-2 nM/sample, depending on run) based on the mean size of each library. The final diluted 8-9 pM pool was denatured using sodium hydroxide according to the Illumina MiSeq protocol and $1 \%$ denatured PhiX beads were added as a sequencing control. Samples were loaded into a 300-cycle MiSeq cartridge and sequenced This protocol is also amenable to the updated Illumina 500 and 600 cycle MiSeq kits. 


\section{Data analysis}

Data was analyzed using Geneious version 5.6 software. A HXB2 reference sequence (GenBank accession \# NC_001802) was uploaded into Geneious and all drug resistance mutations were manually annotated onto the sequence to generate an annotated reference sequence for reference-based assembly (https://dholk.primate.wisc.edu/ wiki/dho/public/page.view?name=default). Sequences are demultiplexed automatically on the MiSeq as part of the data processing steps and two paired .fastq files are generated for each sample representing the two paired-end reads. After importing the fastq files from the MiSeq into Geneious, the two sequence lists from each sample were paired. Next, all sequences were trimmed at the ends as part of the assembly process using the modified-Mott algorithm and quality scores assigned by the sequencing base caller. This algorithm trims the ends to the point where trimming no longer improves the error rate by more than the error probability limit threshold set, in this case 0.001 , or a $0.1 \%$ error rate. Sequences were then mapped to the annotated reference sequence with the following parameters: Gaps were allowed with a maximum of $15 \%$ per read and gap size of 15 , word length was set to 14 , maximum mismatches per read were set to $25 \%$, minimum overlap identity was set to $80 \%$, maximum ambiguity was set to 16 and "search more thoroughly for poor matching reads" was selected. In general, the reference assembler uses a seed and expand-type mapper, followed by a fine-tuning step that was set to none (fast / read mapping) for this analysis. All nucleotide variants represented by at least 5 sequencing reads and at a frequency $>1 \%$ from the reference sequence were then called using the variant finder. This threshold was set based on previous work to establish minimum sequencing read coverage for next-generation sequencing $[22,30]$. To ensure that variants were called relative to the correct codon, the "merge adjacent variations" and "use separate annotations for each variant at a position" were selected. Geneious developed a variant finder analysis tool that allows amino acid variants within a codon to be called together when present on the same sequence to accurately reflect the effects of all single nucleotide changes on the amino acid sequence. This variant finder was used as a plugin with Geneious 5.6 at the time of this analysis but has since been incorporated into Geneious starting with version 6.1 and is now part of their standard Geneious Pro software. Variants and their frequencies were exported into an excel document and filtered for those present in amino acid sites known to correlate with drug resistance based on the Stanford drug resistance database list and the 2012 list of IAS-USA HIV drug resistance mutations as annotated on the reference sequence. Frequency of each variant and the number of sequences representing each nucleotide position containing a variant away from the reference sequence was also calculated by the variant finder plugin.

\section{Phylogenetic analysis}

A consensus sequence was constructed from the referencebased assemblies performed on each sample using the $50 \%$ strict setting. These nucleotide sequences were aligned by the CLUSTAL algorithm in MEGA 6 [43]. A maximum likelihood (ML) phylogenetic analysis was conducted based on the GTR + G+I model, which was chosen using the Bayes Information Criterion in MEGA 6. The reliability of the clustering patterns in ML trees was tested by bootstrapping; 1000 bootstrap pseudo-samples were used.

\section{Additional files}

\section{Additional file 1: $1.0 \%$ agarose gel image of RT-PCR products amplified with universal primers from 19 NIH HIV isolates.}

Additional file 2: All non-synonymous variants detected while sequencing HXB2 clonal stock viruses from three independent PCR amplifications and two independent sequencing reactions to determine the error rates associated with this sequencing method.

Additional file 3: Patient information from samples collected in the ALIVE cohort.

Additional file 4: Maximum likelihood phylogenetic analysis of the assembled sequences from the ALIVE cohort with reference sequences representing subtypes $A, B, C, D, C R F 01 \_A E$ and CRF02_AG using the Bayes Information Criterion in MEGA6.

Additional file 5: External and nested primers used to RT-PCR amplify HIV.

\section{Competing interests}

The authors declare that they have no competing interests.

\section{Authors' contributions}

DMD designed and carried out the drug resistance genotyping assay, analyzed data and wrote the manuscript. ALB participated in data analysis ALH performed phylogenetic analysis. RPW provided the primary samples, helped design the experiments, and initiated the drug resistance genotyping of the ALIVE cohort. GDK and SHM are Co-PIs of the ALIVE study who designed the ALIVE study and oversaw data collection. DHO helped conceive and design the drug resistance genotyping assay. All authors read and approved the manuscript.

\section{Acknowledgments}

Funding for this study was provided through the National Institutes of Health/National Institute on Drug Abuse (Grants: K23DA032306; U01DA023832; R01DA04334; and R01DA12568). We acknowledge the NIH AIDS Research and Reference Reagent Program, Division of AIDS, NIAID, NIH for the International panel of 60 HIV-1 isolates (cat. \# 11412) from Robert Gallo, Smita Kularni, UNAIDS, Nelson Michael, and Victoria Polonis and pHXBn-PLAP-IRES-N+ plasmid (cat. \#3610) from Dr. Benjamin K. Chen and Dr. David Baltimore. We thank Matt Kearse and Geneious for developing the variant finder plugin. We thank Jacquie Astemborski for help locating samples and generating the dataset for the samples used in this study.

\section{Author details}

'Department of Pathology and Laboratory Medicine, University of Wisconsin School of Medicine and Public Health, Madison, WI, USA. ${ }^{2}$ Department of Epidemiology, Epidemiology and Oncology, Johns Hopkins University, Baltimore, MD, USA. ${ }^{3}$ Department of Biology, University of South Carolina, Columbia, South Carolina, USA. ${ }^{4}$ Departments of Medicine, Epidemiology and Oncology, Johns Hopkins University, Baltimore, MD, USA. ${ }^{5}$ Department of Medicine, Division of Infectious Disease, University of Wisconsin School of Medicine and Public Health, Madison, WI, USA. 
Received: 3 April 2014 Accepted: 3 December 2014

Published online: 23 December 2014

\section{References}

1. Choopanya K, Martin M, Suntharasamai P, Sangkum U, Mock PA Leethochawalit M, Chiamwongpaet S, Kitisin P, Natrujirote P, Kittimunkong S, Chuachoowong R, Gvetadze RJ, McNicholl JM, Paxton LA, Curlin ME, Hendrix CW, Vanichseni S: Antiretroviral prophylaxis for HIV infection in injecting drug users in Bangkok, Thailand (the Bangkok Tenofovir Study): a randomised, double-blind, placebo-controlled phase 3 trial. Lancet 2013, 381:2083-2090.

2. Cohen MS, Chen YQ, McCauley M, Gamble T, Hosseinipour MC, Kumarasamy N, Hakim JG, Kumwenda J, Grinsztejn B, Pilotto JH, Godbole SV, Mehendale S, Chariyalertsak S, Santos BR, Mayer KH, Hoffman IF, Eshleman SH, Piwowar-Manning E, Wang L, Makhema J, Mills LA, de Bruyn G, Sanne I, Eron J, Gallant J, Havlir D, Swindells S, Ribaudo H, Elharrar V, Burns D, et al: Prevention of HIV-1 infection with early antiretroviral therapy. N Engl J Med 2011, 365:493-505.

3. van de Vijver DA, Nichols BE, Abbas UL, Boucher CA, Cambiano V, Eaton JW, Glaubius R, Lythgoe K, Mellors J, Phillips A, Sigaloff KC, Hallett TB: Preexposure prophylaxis will have a limited impact on HIV-1 drug resistance in sub-Saharan Africa: a comparison of mathematical models. AIDS 2013, 27(18):2943-2951.

4. Abbas UL, Glaubius R, Mubayi A, Hood G, Mellors JW: Antiretroviral therapy and pre-exposure prophylaxis: combined impact on HIV transmission and drug resistance in South Africa. J Infect Dis 2013, 208:224-234.

5. Jordan MR, Bennett DE, Wainberg MA, Havlir D, Hammer S, Yang C, Morris L, Peeters M, Wensing AM, Parkin N, Nachega JB, Phillips A, De Luca A, Geng E, Calmy A, Raizes E, Sandstrom P, Archibald CP, Perriens J, McClure CM, Hong SY, McMahon JH, Dedes N, Sutherland D, Bertagnolio S: Update on world health organization HIV drug resistance prevention and assessment strategy: 2004-2011. Clin Infect Dis 2012, 54(Suppl 4):S245-S249.

6. De Luca A, Hamers RL, Schapiro JM: Antiretroviral treatment sequencing strategies to overcome HIV type 1 drug resistance in adolescents and adults in low-middle-income countries. J Infect Dis 2013, 207(Suppl 2):S63-S69.

7. Dudley DM, Chin EN, Bimber BN, Sanabani SS, Tarosso LF, Costa PR, Sauer MM, Kallas EG, O'Connor DH: Low-cost ultra-wide genotyping using Roche/454 pyrosequencing for surveillance of HIV drug resistance. PLoS One 2012, 7:e36494.

8. DHHS: Recommendation on Integrase Inhibitor Use in Antiretroviral Treatment-Naive HIV-Infected Individuals from the HHS Panel on Antiretroviral Guidelines for Adults and Adolescents; 2013

9. Johnson LB, Saravolatz LD: The quad pill, a once-daily combination therapy for HIV infection. Clin Infect Dis 2014, 58:93-98.

10. Van den Eede $P$, Van Wesenbeeck $L$, Verlinden $Y$, Feyaerts $M$, Smits $V$, Verheyen A, Vanhooren L, Deloof A, Villacian J, Pattery T: HIV-1 genotyping of the protease-reverse transcriptase and integrase genes to detect mutations that confer antiretroviral resistance. Methods Mol Biol 2013, 1030:37-55.

11. Eshleman SH, Hudelson SE, Smith P, Hackett J, Holzmayer V, Swanson P, Devare SG, Marlowe N: Analysis of pol integrase sequences in diverse HIV type 1 strains using a prototype genotyping assay. AIDS Res Hum Retroviruses 2009, 25:343-345.

12. Eshleman SH, Hackett JJ, Swanson P, Cunningham SP, Drews B, Brennan C, Devare SG, Zekeng L, Kaptue L, Marlowe N: Performance of the Celera diagnostics ViroSeq HIV-1 genotyping system for sequence-based analysis of diverse human immunodeficiency virus type 1 strains. J Clin Microbiol 2004, 42:2711-2717

13. Hemelaar J, Gouws E, Ghys PD, Osmanov S: Global trends in molecular epidemiology of HIV-1 during 2000-2007. AIDS 2011, 25:679-689.

14. Aghokeng AF, Mpoudi-Ngole E, Chia JE, Edoul EM, Delaporte E, Peeters M: High failure rate of the ViroSeq HIV-1 genotyping system for drug resistance testing in Cameroon, a country with broad HIV-1 genetic diversity. J Clin Microbiol 2011, 49:1635-1641.

15. Aitken SC, Kliphuis A, Wallis CL, Chu ML, Fillekes Q, Barth R, Stevens W, de Wit TF R, Schuurman R: Development and evaluation of an assay for HIV-1 protease and reverse transcriptase drug resistance genotyping of all major group-M subtypes. J Clin Virol 2012, 54:21-25.

16. Zhou Z, Wagar N, DeVos JR, Rottinghaus E, Diallo K, Nguyen DB, Bassey O, Ugbena R, Wadonda-Kabondo N, McConnell MS, Zulu I, Chilima B, Nkengasong J, Yang C: Optimization of a low cost and broadly sensitive genotyping assay for HIV-1 drug resistance surveillance and monitoring in resource-limited settings. PLOS One 2011, 6:e28184.

17. Wallis CL, Papathanasopoulos MA, Lakhi S, Karita E, Kamali A, Kaleebu P, Sanders E, Anzala O, Bekker LG, Stevens G, de Wit TF, Stevens W: Affordable in-house antiretroviral drug resistance assay with good performance in non-subtype B HIV-1. J Virol Methods 2010, 163:505-508.

18. Acharya A, Vaniawala S, Shah P, Misra RN, Wani M, Mukhopadhyaya PN: Development, validation and clinical evaluation of a low cost in-house HIV-1 drug resistance genotyping assay for Indian patients. PLOS One 2014, 9:e105790.

19. Pou C, Noguera-Julian M, Perez-Alvarez S, Garcia F, Delgado R, Dalmau D, Alvarez-Tejado M, Gonzalez D, Sayada C, Chueca N, Pulido F, Ibanez L, Rodriguez C, Casadella M, Santos JR, Ruiz L, Clotet B, Paredes R: Improved prediction of salvage antiretroviral therapy outcomes using ultrasensitive HIV-1 drug resistance testing. Clin Infect Dis 2014, 59:578-588.

20. Manasa J, Danaviah S, Pillay S, Padayachee P, Mthiyane H, Mkhize C, Lessells RJ, Seebregts C, de Wit TF, Viljoen J, Katzenstein D, De Oliveira T: An affordable HIV-1 drug resistance monitoring method for resource limited settings. J Vis Exp 2014, (85): doi: 10.3791/51242.

21. Simen BB, Braverman MS, Abbate I, Aerssens J, Bidet Y, Bouchez O, Gabriel C, Izopet J, Kessler HH, Stelzl E, Di Giallonardo F, Schlapbach R, Radonic A, Paredes R, Recordon-Pinson P, Sakwa J, St John EP, Schmitz-Agheguian GG, Metzner KJ, Daumer MP: An international multicenter study on HIV-1 drug resistance testing by 454 ultra-deep pyrosequencing. J Virol Methods 2014, 204:31-37.

22. Wang C, Mitsuya Y, Gharizadeh B, Ronaghi M, Shafer RW: Characterization of mutation spectra with ultra-deep pyrosequencing: application to HIV-1 drug resistance. Genome Res 2007, 17:1195-1201.

23. Church JD, Jones D, Flys T, Hoover D, Marlowe N, Chen S, Shi C, Eshleman JR, Guay LA, Jackson JB, Kumwenda N, Taha TE, Eshleman SH: Sensitivity of the ViroSeq HIV-1 genotyping system for detection of the $\mathrm{K} 103 \mathrm{~N}$ resistance mutation in HIV-1 subtypes A, C, and D. J Mol Diagn 2006, 8:430-432.

24. Loman NJ, Misra RV, Dallman TJ, Constantinidou C, Gharbia SE, Wain J, Pallen MJ: Performance comparison of benchtop high-throughput sequencing platforms. Nat Biotechnol 2012, 30:434-439.

25. Quail MA, Smith M, Coupland P, Otto TD, Harris SR, Connor TR, Bertoni A, Swerdlow HP, GU Y: A tale of three next generation sequencing platforms: comparison of Ion Torrent, Pacific Biosciences and Illumina MiSeq sequencers. BMC Genomics 2012, 13:341.

26. Allhoff M, Schonhuth A, Martin M, Costa IG, Rahmann S, Marschall T: Discovering motifs that induce sequencing errors. BMC Bioinformatics 2013, 14(Suppl 5):S1.

27. Shao W, Boltz VF, Spindler JE, Kearney MF, Maldarelli F, Mellors JW, Stewart C, Volfovsky N, Levitsky A, Stephens RM, Coffin JM: Analysis of 454 sequencing error rate, error sources, and artifact recombination for detection of Low-frequency drug resistance mutations in HIV-1 DNA. Retrovirology 2013, 10:18.

28. Vlahov D, Anthony JC, Munoz A, Margolick J, Nelson KE, Celentano DD, Solomon L, Polk BF: The ALIVE study, a longitudinal study of HIV-1 infection in intravenous drug users: description of methods and characteristics of participants. NIDA Res Monogr 1991, 109:75-100.

29. Westergaard RP, Kirk GD, Richesson DR, Galai N, Mehta SH: Incarceration predicts virologic failure for HIV-infected injection drug users receiving antiretroviral therapy. Clin Infect Dis 2011, 53:725-731.

30. Gall A, Ferns B, Morris C, Watson S, Cotten M, Robinson M, Berry N, Pillay D, Kellam P: Universal amplification, next-generation sequencing, and assembly of HIV-1 genomes. J Clin Microbiol 2012, 50:3838-3844.

31. Gibson RM, Meyer AM, Winner D, Archer J, Feyertag F, Ruiz-Mateos E, Leal M, Robertson DL, Schmotzer CL, Quinones-Mateu ME: Sensitive deep sequencing-based HIV-1 genotyping assay to simultaneously determine susceptibility to protease, reverse transcriptase, integrase, and maturation inhibitors, as well as HIV-1 coreceptor tropism. Antimicrob Agents Chemother 2014, 58(4):2167-2185.

32. Hedskog C, Mild M, Jernberg J, Sherwood E, Bratt G, Leitner T, Lundeberg J, Andersson B, Albert J: Dynamics of HIV-1 quasispecies during antiviral treatment dissected using ultra-deep pyrosequencing. PLoS One 2010, 5:e11345.

33. Bimber BN, Dudley DM, Lauck M, Becker EA, Chin EN, Lank SM, Grunenwald HL, Caruccio NC, Maffitt M, Wilson NA, Reed JS, Sosman JM, Tarosso LF, Sanabani S, Kallas EG, Hughes AL, O'Connor DH: Whole-genome characterization of human and simian immunodeficiency virus intrahost diversity by ultradeep pyrosequencing. J Virol 2010, 84:12087-12092. 
34. Rabi SA, Laird GM, Durand CM, Laskey S, Shan L, Bailey JR, Chioma S, Moore RD, Siliciano RF: Multi-step inhibition explains HIV-1 protease inhibitor pharmacodynamics and resistance. J Clin Invest 2013, 123:3848-3860.

35. Sutherland KA, Mbisa JL, Cane PA, Pillay D, Parry CM: Contribution of Gag and protease to variation in susceptibility to protease inhibitors between different strains of subtype $B$ human immunodeficiency virus type 1. J Gen Virol 2014, 95:190-200.

36. Ghosn J, Delaugerre C, Flandre P, Galimand J, Cohen-Codar I, Raffi F, Delfraissy JF, Rouzioux C, Chaix ML: Polymorphism in Gag gene cleavage sites of HIV-1 non-B subtype and virological outcome of a first-line lopinavir/ritonavir single drug regimen. PLoS One 2011, 6:e24798.

37. Rhee SY, Gonzales MJ, Kantor R, Betts BJ, Ravela J, Shafer RW: Human immunodeficiency virus reverse transcriptase and protease sequence database. Nucleic Acids Res 2003, 31:298-303.

38. Shafer RW: Rationale and uses of a public HIV drug-resistance database. $J$ Infect Dis 2006, 194(Suppl 1):S51-S58.

39. Administration USFaD: FDA allows marketing of four "next generation" gene sequencing devices. FDA news release, 2013. Department of Health and Human Services website: http://www.fda.gov/NewsEvents/Newsroom/ PressAnnouncements/ucm375742.htm.

40. Westergaard RP, Hess T, Astemborski J, Mehta SH, Kirk GD: Longitudinal changes in engagement in care and viral suppression for HIV-infected injection drug users. AIDS 2013, 27:2559-2566.

41. Salter ML, Lau B, Go VF, Mehta SH, Kirk GD: HIV infection, immune suppression, and uncontrolled viremia are associated with increased multimorbidity among aging injection drug users. Clin Infect Dis 2011, 53:1256-1264

42. Vlahov D, Galai N, Safaeian M, Galea S, Kirk GD, Lucas GM, Sterling TR: Effectiveness of highly active antiretroviral therapy among injection drug users with late-stage human immunodeficiency virus infection. Am J Epidemiol 2005, 161:999-1012.

43. Tamura K, Peterson D, Peterson N, Stecher G, Nei M, Kumar S: MEGA5: molecular evolutionary genetics analysis using maximum likelihood, evolutionary distance, and maximum parsimony methods. Mol Biol Evol 2011, 28:2731-2739

\section{Submit your next manuscript to BioMed Central and take full advantage of:}

- Convenient online submission

- Thorough peer review

- No space constraints or color figure charges

- Immediate publication on acceptance

- Inclusion in PubMed, CAS, Scopus and Google Scholar

- Research which is freely available for redistribution 